SEPA

\title{
Jetzt Kombimandat einholen
}

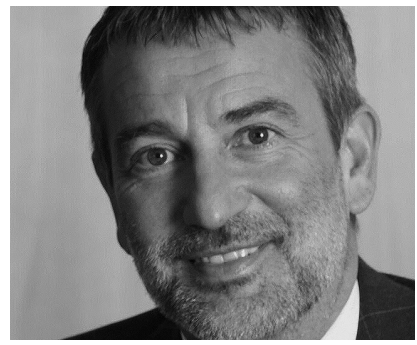

VON BERND BAUER

Bernd Bauer ist Direktor Informationstechnologie bei der Bank für Sozialwirtschaft AG.

E-Mail b.bauer@sozialbank.de

\author{
Bis voraussichtlich November 2013 sollen die nationalen \\ Zahlungsverkehrssysteme durch europaweit \\ standardisierte Verfahren abgelöst werden. Wer Kosten \\ vermeiden und nicht unter Zeitdruck geraten will, sollte \\ bereits jetzt mit vorbereitenden Umstellungsmaß- \\ nahmen beginnen.
}

Im Rahmen der Realisierung des Binnenmarktes der Europäischen Union wird ein einheitlicher Euro-Zahlungsverkehrsraum geschaffen: »Single European Payment Area «, kurz SEPA. Darin wird nicht mehr zwischen nationalen und grenzüberschreitenden Zahlungen unterschieden. Bisher verfügt jedes Land im Euro-Raum über eigene technische Standards, beispielsweise bei der Kontonummern-Systematik, dem Datenformat für den Zahlungsaustausch oder der Gestaltung einzelner Zahlungsverfahren.

SEPA wird diese traditionellen Strukturen aufbrechen: Nutzer von Zahlungsverkehrsdienstleistungen werden im SEPA-Verfahren künftig von einer beliebigen Bank aus bargeldlose Euro-Zahlungen im ganzen Euroraum tätigen und hierbei einheitliche Zahlungsinstrumente - SEPA-Überweisung (»Credit Transfer «), SEPA-Lastschrift (»Direct Debit «) und SEPA-Kartenzahlungen ( Cards Framework «) - einsetzen können. Alle Zahlungen werden behandelt wie nationale Zahlungen, mit einer garantierten Ausführungszeit bis zur Kontogutschrift von maximal drei Bankarbeitstagen (ab 1. Januar 2012: ein Bankarbeitstag). Teilnehmerländer an der SEPA sind die 27 Mitgliedsstaaten der Europäischen Union sowie Island, Liechtenstein, Norwegen und die Schweiz. Alle Transaktionen werden in Euro durchgeführt.

Die Banken sind zunächst angehalten, die SEPA-Zahlungsinstrumente zusätzlich zu den nationalen Zahlungsverkehrssystemen anzubieten. Wie lange die nationalen Zahlungsverkehrssysteme parallel zu den SEPA-Verfahren angeboten werden können, steht noch nicht verbindlich fest. Erwartet wird die endgültige Umstellung frühestens $a b$ November 2012. Damit diese reibungslos funktioniert, sind in allen sozialen Organisationen und Unternehmen vorbereitende organisatorische und technische Maßnahmen erforderlich.

Statt Bankleitzahl und Kontonummer ist zur Identifizierung des Zahlungsempfängers künftig erforderlich:

- die IBAN = »International Bank Account Number «

- und der BIC $=$ »Bank Identifier Code $«$.

Konkret bedeutet das: Allein die IBAN zählt, der Name des Zahlungsempfängers ist also nicht mehr wichtig. Es gibt keine Namensüberprüfung seitens der Banken mehr. Die IBAN ist eine international standardisierte Kontonummer, bestehend aus insgesamt 22 Stellen. Der BIC (auch bekannt als »SWIFT-Code «) ist ein international standardisierter Bankcode, nach dem weltweit jedes Kreditinstitut eindeutig identifiziert werden kann. Der BIC besteht aus acht bis elf Stellen.

Während die Einführung der SEPAÜberweisung (SEPA-CT) und der SEPAKartenzahlung (SEPA-CF) im Januar 2008 mit vereinheitlichten Standards gelaufen ist, entschied sich der Europäische Zahlungsverkehrsrat, der die Einführung 
der SEPA steuert, für die Einführung der SEPA-Lastschrift (SEPA-DD), die seit November 2009 läuft, aufgrund der unterschiedlichen rechtlichen Rahmenbedingungen und verschiedenen nationalen Lastschriftverfahren gegen eine Harmonisierung der bestehenden nationalen Lastschriftverfahren.

Ein völlig neues Einzugsverfahren für die SEPA-DD wurde entwickelt. Die SEPA-DD unterscheidet sich in mehreren Punkten von der heutigen deutschen Lastschrift: Der Zahlungspflichtige erteilt dem Gläubiger keine Einzugsermächtigung mehr, sondern ein Mandat. Dieses ermächtigt den Zahlungsempfänger nicht nur zum Einzug der Zahlung, sondern beauftragt das kontoführende Institut, die übermittelte Lastschriftanweisung zu erfüllen und das entsprechende Konto zu belasten. Alle Mandate sind vom Kontoinhaber eigenhändig zu unterzeichnen. Die Widerspruchsfrist für den Zahlungspflichtigen beträgt bis zu acht Wochen nach der Kontobelastung. Zudem wird künftig unterschieden zwischen einer SEPA-Basislastschrift (»SEPA Core Direct Debit «) und einer SEPA-Firmenlastschrift (»SEPA Busines to Bussines Direct Debit «).

Neu sind auch fest definierte Vorlauffristen für die Vorlage der Lastschrift an die Zahlstelle: Die erste Inkassostelle muss die Lastschrift so rechtzeitig an die Zahlstelle weiterleiten, dass sie spätestens fünf Tage bei Erstlastschriften und zwei Tage bei Folgelastschriften vor Fälligkeit vorliegt.

Spenden- und Mitgliedsorganisationen, die künftig die SEPA-DD einsetzen wollen, müssen ihre insgesamt circa zehn bis zwölf Millionen Dauerlastschriften auf das neue Mandat umstellen. Das heißt: Alle derzeitigen Spender und Mitglieder müssen zur Umstellung auf das Lastschriftmandat aufgefordert werden. Befürchtet wird, dass zahlreiche Spender oder Mitglieder die Gelegenheit nutzen werden, ihre Lastschriften auslaufen zu lassen.

Außerdem wird circa 90 Prozent des Internet-Spendenzahlungsverkehrs in
Deutschland zurzeit mittels Lastschrift ohne " physikalische Unterschrift « abgewickelt. Und im Kontext von Spendengalas im Fernsehen werden Lastschriften mit erheblichen Summen erteilt. Mit der Einführung der SEPA-DD in der vorgesehenen Form ist dies nicht mehr möglich.

Als vorbereitende Maßnahme bis zur endgültigen Einführung der SEPA-DD empfiehlt die Bank für Sozialwirtschaft ab sofort die Nutzung eines Kombimandates, das bereits im jetzigen Lastschrifteinzugsverfahren, aber auch im künftigen SEPA-DD Verfahren eingesetzt werden kann. Beim Kombimandat wird die Einzugsermächtigung erteilt und zugleich bereits ein SEPA-Lastschriftmandat vergeben. Außerdem sollte jedes Unternehmen mit seinem Software-Hersteller abklären, bis zu welchem Zeitpunkt die technische Umstellung seiner Systeme auf das XML Format erfolgt. Als Datenformat für SEPA-CT und SEPA-DD wird ein XMLbasierter, von S.W.I.F.T. entwickelter ISO-Standard (ISO 20022) genutzt.

\section{Dinge, die Sie jetzt tun müssen: Checkliste zur SEPA-Einführung}

\section{Organisatorische Maßnahmen}

1 Benennen Sie einen SEPA-Beauftragten oder einen - SEPA-Ansprechpartner in Ihrer Organisation.

7 Entwickeln Sie einen individuellen Zeit- und Umset2. zungszeitplan für Ihre Organisation.

3 Analysieren Sie Ihre Zahlungsströme und die Struktur 3. Ihres Zahlungsverkehrs, um mögliche SEPA-Auswirkungen auf Ihre Organisation einschätzen zu können.

1 Setzen Sie sich mit Ihrer Hausbank wegen der Ände- rungen in Verbindung.

5. Geben Sie auf Ihren Briefbögen bereits jetzt Ihre IBAN 2. und BIC an.

6 Beantragen Sie die »Gläubigeridentifikationsnum-

- mer« bei der Deutschen Bundesbank für die Teilnahme am SEPA-Lastschriftverfahren.

7 Überlegen Sie sich eine Systematik für die Mandatsrefe- renznummern und pflegen diese in IhreStammdaten ein.

P Prüfen Sie, ob Ihren bisherigen Lastschriften gültige Last8. schrifteinzugsermächtigungen zugrunde liegen, damit diese in ein SEPA-Mandat gewandelt werden können.

Lassen Sie sich von neuen Spendern oder Mitgliedern - ein Kombimandat ausstellen, also eine deutsche Lastschrifteinzugsermächtigung plus SEPA-Mandat.

10. Ergänzen Sie Ihre Spender- und Mitgliederstammdaten um IBAN und BIC.
11 Prüfen Sie, ob die Datenkonvertierung über einen externen Dienstleister durchgeführt werden soll.

\section{Technische Maßnahmen}

17 Testen Sie, ob Ihre Zahlungsverkehrs-Software (Elec2. tronic-banking-Produkte) "SEPA-ready« sind.

12 Prüfen Sie, ob Ihre Finanzbuchhaltung "SEPA-ready“ . ist.

1 Stellen Sie fest, über welche Software-Produkte sonst - noch Zahlungsdateien generiert werden (z. B. Mitglieder-oder Spenderverwaltungsprogramme).

15 Prüfen Sie, in welche Datenbanken SEPA-Datenele5. mente zu integrieren sind.

16 Kontaktieren Sie Ihre Softwareanbieter zu den Umstel- lungsmöglichkeiten und erforderlichen Änderungen.

17.

SEPA-Zahlungen unterstützen nur noch den neuen "Electronic Banking Internet Communication Standard « (EBICS). Dieser löst den bisherigen Standard FTAM vollständig ab. Stellen Sie also rechtzeitig um!

18 Denken Sie daran, dass der Datenträgeraustausch per - Diskette oder CD unter SEPA nicht mehr unterstützt wird.

10 Beachten Sie die Auswirkungen des vergrößerten Da- tenvolumens unter SEPA (XML-Format) auf Ihre Hardware-Konfiguration.

2 Prüfen Sie Ihre Softwareapplikation für Löhne und - Gehälter bezüglich SEPA-Fähigkeit. 